\title{
Current evidence does not support a role for vertebroplasty
}

The authors of this letter (BMJ 2011;343:d5043, doi:10.1136/ bmj.d5043), Rachelle Buchbinder and Paul Glasziou, wish to clarify two points that we inadvertently altered during editing. Firstly, in relation to the first sentence, they say that, although they agree with D J Wilson (reference 1) that vertebroplasty cannot be recommended as first line therapy, current evidence favours no role whatsoever (this should have been be reflected in the title too). Secondly, they say that rather than suggesting that doubts remain about the role of vertebroplasty for osteoporotic vertebral fracture (as stated in the final paragraph), they had wanted to make the point that if others thought additional randomised trials were justified, it would be essential to blind treatment allocation, participants, and investigators, necessitating the use of a sham or placebo control.

Cite this as: BMJ 2011;343:d5389

๑ BMJ Publishing Group Ltd 2011 